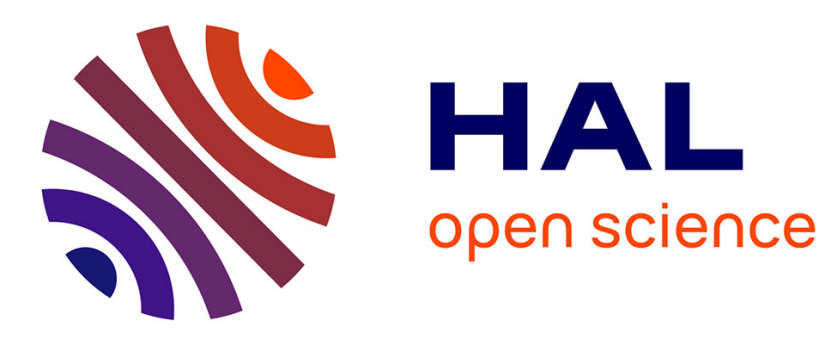

\title{
Vertical transmission of arboviruses in mosquitoes: A historical perspective
}

Sebastian Lequime, Louis Lambrechts

\section{To cite this version:}

Sebastian Lequime, Louis Lambrechts. Vertical transmission of arboviruses in mosquitoes: A historical perspective. Infection, Genetics and Evolution, 2014, 28, pp.681-690. 10.1016/j.meegid.2014.07.025 . pasteur-01976254

\section{HAL Id: pasteur-01976254 \\ https://hal-pasteur.archives-ouvertes.fr/pasteur-01976254}

Submitted on 8 Feb 2019

HAL is a multi-disciplinary open access archive for the deposit and dissemination of scientific research documents, whether they are published or not. The documents may come from teaching and research institutions in France or abroad, or from public or private research centers.
L'archive ouverte pluridisciplinaire HAL, est destinée au dépôt et à la diffusion de documents scientifiques de niveau recherche, publiés ou non, émanant des établissements d'enseignement et de recherche français ou étrangers, des laboratoires publics ou privés.

\section{(ㄷ)(1)}

Distributed under a Creative Commons Attribution| 4.0 International License 


\title{
Vertical transmission of arboviruses in mosquitoes: a historical perspective
}

\author{
Sebastian Lequime ${ }^{1,2 *}$ and Louis Lambrechts ${ }^{1 *}$ \\ ${ }^{1}$ Insect-Virus Interactions Group, Department of Genomes and Genetics, Institut Pasteur- \\ Centre National de la Recherche Scientifique, Unité de Recherche Associée 3012, Paris, \\ France \\ ${ }^{2}$ University Pierre et Marie Curie, Cellule Pasteur UPMC, Paris, France
}

*Corresponding author. Address: G5 Insect-Virus Interactions, Institut Pasteur, 28 rue du Docteur Roux, 75724 Paris Cedex 15, France.

E-mail addresses: sebastian.lequime@pasteur.fr (S. Lequime); louis.lambrechts@pasteur.fr (L. Lambrechts). 


\begin{abstract}
Arthropod-borne viruses (arboviruses) are mainly transmitted horizontally among vertebrate hosts by blood-feeding invertebrate vectors, but can also be transmitted vertically in the vector from an infected female to its offspring. Vertical transmission (VT) is considered a possible mechanism for the persistence of arboviruses during periods unfavorable for horizontal transmission, but the extent and epidemiological significance of this phenomenon have remained controversial. To help resolve this question, we reviewed over a century of published literature on VT to analyze historical trends of scientific investigations on experimental and natural occurrence of VT in mosquitoes. Our synthesis highlights the influence of major events of public health significance in arbovirology on the number of VT publications. Epidemiological landmarks such as emergence events have significantly stimulated VT research. Our analysis also reveals the association between the evolution of virological assays and the probability of VT detection. Increased sensitivity and higherthroughput of modern laboratory assays resulted in enhanced VT detection. In general, VT contribution to arbovirus persistence is likely modest because vertically infected mosquitoes are rarely observed in nature. Taken together, however, our results call for caution when interpreting VT studies because their conclusions are context- and method-dependent.
\end{abstract}

Keywords: Arbovirus; mosquito; vertical transmission; systematic review; historical trend. 


\section{Highlights}

- The extent and epidemiological importance of arbovirus VT are still debated.

- Arboviral emergence events have stimulated VT research.

- More recent laboratory assays are associated with enhanced VT detection.

- Typically $<0.1 \%$ of mosquitoes are vertically infected in nature.

- The outcome of VT studies is context- and method-dependent. 


\section{Introduction}

According to the United States Center for Disease Control and Prevention (CDC) Arbovirus Catalog (Centers for Disease Control and Prevention, 2010), there are currently at least 530 identified arthropod-borne viruses (arboviruses), of which about a hundred cause human disease. Among them, four major viral genera account for the majority of arboviral diseases: Flavivirus (e.g., dengue, West Nile, Japanese encephalitis, and yellow fever viruses), Alphavirus (e.g., chikungunya, Eastern equine encephalomyelitis, Western equine encephalomyelitis and Venezuelan equine encephalitis viruses), Orthobunyavirus (e.g., California encephalitis and LaCrosse viruses) and Phlebovirus (e.g., Rift Valley fever and sandfly fever viruses).

During the past few decades, several arboviruses have emerged globally and are now considered among the most important public health concerns for the $21^{\text {st }}$ century (Gubler, 2002). Dengue, for example, has become the most prevalent arthropod-borne viral disease of humans over the last few decades (Messina et al., 2014); it has recently been estimated that there are 390 million human dengue infections each year (Bhatt et al., 2013). With only a few licensed vaccines and virtually no therapeutics available, antivectorial measures are often the only way to prevent arboviral diseases. Historically, however, the implementation of vector control measures has generally been difficult to sustain.

Arboviruses are naturally maintained in a transmission cycle between vertebrate and arthropod hosts (Gubler, 2001). The majority of arthropod hosts, generally referred to as vectors, are blood-feeding mosquitoes. Rather than a simple alternation within a single hostvector pair, arbovirus transmission often occurs through highly complex transmission networks that include various hosts and vectors (Diaz et al., 2012). Humans in particular, are not necessarily at the center of the transmission network and may only be incidental hosts 
(e.g., West Nile virus). Whereas some host or vector species are central to epidemic arbovirus transmission, others can be part of alternative transmission pathways, participating in the maintenance of the virus in nature during inter-epidemic periods. As an example, for some authors, fox squirrels may contribute to alternative transmission of West Nile virus in suburban communities (Root et al., 2006; 2007). In many regions of the world, climatic conditions do not allow mosquito reproductive activity all year long. During the dry season in tropical areas or the cold season in temperate regions, the absence or low density of adult mosquitoes is unlikely to support continuous host-to-vector (horizontal) transmission (Leake, 1984). Survival of mosquitoes during dry and cold seasons involves different physiological and/or behavioral mechanisms that may impact virus transmission differently. Besides, arboviral infections in vertebrate hosts typically produce a short-lived viremic period that eventually results in immunization of the host. A high level of herd immunity in the host community may thus prevent transmission above the minimum level required for sustained horizontal transmission. The existence of reservoir host species, alternative transmission mechanism or virus re-introduction, have been proposed to explain the maintenance of arboviruses during unfavorable periods or when herd immunity is high (for review, see Reeves, 2004).

One popular hypothesis to explain the persistence of arboviruses during unfavorable periods is the occurrence of vertical transmission (VT) in the arthropod vector. In this article, we define VT as the transmission of an arbovirus from an infected female mosquito to its offspring, regardless of the underlying mechanism. VT may occur through two main mechanisms. Transovarial transmission (TOT) occurs when the virus infects the germinal tissues of the female mosquito, whereas trans-egg VT takes place during oviposition in the fully formed egg (Rosen, 1988). TOT typically achieves a higher efficiency of VT than trans- 
egg mechanisms especially when the germ cells are permanently infected so that most of the offspring are infected in the following generation (Tesh, 1984).

Under the VT scenario, the arbovirus present in the mosquito eggs, larvae or adults, including nulliparous females entering diapause, may survive throughout the unfavorable period without the need for a vertebrate host. Such a mixed-mode transmission (i.e., both horizontal and VT) is widespread among symbionts across taxa (Ebert, 2013). Here symbiosis is defined as any type of persistent biological interaction, which includes mutualistic, commensalistic and antagonistic relationships. Although the infection cost is often modest to the vector, arboviruses are considered parasites of mosquitoes (Lambrechts and Scott, 2009). Combining horizontal with VT enlarges considerably the range of ecological conditions in which a symbiont can persist. In host species with diapause or discrete generations, VT may allow the symbiont to endure periods when horizontal transmission is not possible. Trade-offs between the two modes of transmission have been documented but are not universal (Ebert, 2013). In addition to ecological factors that may favor horizontal over VT (e.g., climate), theory suggests that VT should be reduced in arboviruses with complex transmission networks because horizontal transmission among genetically disparate hosts hinders coadaptation between vertically transmitted viruses and their hosts.

Both the very existence and the epidemiological significance of arbovirus VT have remained controversial since it was first suggested in the scientific literature over a century ago, at the onset of arbovirology. Carlos Finlay, who first introduced the idea of vectorial transmission of yellow fever virus by mosquitoes in 1881, extended his theory in 1899 , suggesting that the yellow fever agent could be transmitted by an infected mosquito to its progeny (Finlay, 1899). During their investigations in Cuba, the Yellow Fever U.S. Army Commission proved Carlos Finlay's original theory right in 1901 and experimentally tested, albeit unsuccessfully, the possibility of yellow fever virus VT in mosquitoes (Reed et al., 
1901). The same year, the Cuban physician Juan Guiteras also failed to succeed in demonstrating yellow fever virus VT (Guiteras, 1901).

Between 1901 and 1905, a group of French scientists from the Pasteur Institute carried out studies on yellow fever in Rio de Janeiro, Brazil. Among them, Paul-Louis Simond and Emile Marchoux finally demonstrated the VT hypothesis. They stated, however, that in their opinion the phenomenon was certainly infrequent (Marchoux and Simond, 1906; 1905). Following this discovery, many have tried to replicate the experiment, but none have succeeded (Davis and Shannon, 1930; Frobisher et al., 1931; Hindle, 1930; Rosenau and Goldberger, 1906).

The possibility of VT of other arboviruses was also investigated during the first half of the $20^{\text {th }}$ century. Whereas early studies on dengue virus failed to provide evidence of VT (Siler et al., 1926; Simmons et al., 1931), a Japanese team demonstrated VT of Japanese encephalitis virus (Mitamura et al., 1939), but their work, published in German in a Japanese journal on the eve of World War II, went unnoticed by the scientific community. Studies on VT resumed in the 1950s and 1960s with a team from the Communicable Disease Center (ancestor of CDC) that was investigating viruses responsible for encephalitis (alphaviruses, such as Eastern equine encephalomyelitis, Western equine encephalomyelitis or Venezuelan equine encephalitis viruses; flaviviruses, such as St. Louis encephalitis virus). Their conclusions, however, were inconsistent with both positive (Chamberlain et al., 1956b; Kissling et al., 1956; 1957) and negative results (Chamberlain et al., 1956a; Chamberlain and Sudia, 1957; Chamberlain et al., 1959). The outcome of these studies did not seem to depend on the virus under consideration.

In 1972, Robert B. Tesh and colleagues provided a clear demonstration of vesicular stomatitis virus VT in Lutzomyia sandflies (Tesh et al., 1972). The next year, another team led by Douglas M. Watts, published evidence of VT for LaCrosse virus (Orthobunyavirus) in 
experimentally infected Aedes triseriatus mosquitoes (Watts et al., 1973). Research on arbovirus VT by mosquito vectors has been vigorous ever since (Fig. 1), although a closer look reveals considerable heterogeneity associated with historical events and the evolution of laboratory assays used to detect VT experimentally or in a natural setting.

In the present study, we conducted a systematic review of over a century of published literature on arbovirus VT to analyze quantitatively historical trends of research on experimental and natural occurrence of VT in mosquitoes. 


\section{Material and methods}

\subsection{Literature search}

Between 22 June 2011 and 25 September 2013, a systematic literature search was conducted in NCBI PubMed, ISI Web of Science, Armed Forces Pest Management Board Literature Retrieval System and Pasteur Institute Media Library. Citations in the identified articles were also examined individually in order to recover additional references. When the article was not found using the databases mentioned above, the corresponding authors and/or journal were contacted to obtain a copy of the publication. Older publications were found through Internet Archive (http://www.archive.org/).

Publications were searched regardless of their language, including English, French, German, Japanese and Chinese. Japanese and Chinese publications without a full abstract in English were excluded for practical reasons.

The review focused on arboviruses transmitted by mosquitoes and therefore articles dealing with VT in ticks or other arthropods were excluded. Likewise, publications about insect-specific viruses were excluded. Within mosquito-borne arboviruses, the review was restricted to VT in three main arboviral families: Bunyaviridae, Flaviviridae and Togaviridae.

\subsection{Databases}

Three databases were created in MySQL using the Sequel Pro® software. Contents of the three databases are described below.

2.2.1. Historical trends. To analyze historical trends in VT research, a first database (database \#1) was built that contains all publications related to VT according to the inclusion criteria indicated above. For each individual publication contained in this database, basic bibliographical data, virus taxonomy and study type were recorded. Study type consisted of 
five categories: 'Experimental' studies looked for evidence of VT based on laboratory experiments under controlled environments; 'Natural' studies attempted to demonstrate VT in nature, by collecting immature, male or overwintering female mosquitoes; 'Both' are studies that conducted both experimental and natural investigations; 'Modeling' studies used mathematical models to evaluate the potential role of VT in arbovirus epidemiology; 'Reviews \& Opinion' articles synthesized earlier work and/or commented the possibility of VT.

2.2.2. Evolution of virological assays. In order to examine how the evolution of laboratory assays in virology influenced the outcome of VT studies, two additional databases were assembled. Database \#2 consists of 'experimental' studies and database \#3 consists of 'natural' studies. Both were derived from database \#1, but were restricted to the 'experimental', 'natural', and 'both' types of studies. Additionally, inclusion criteria were more stringent for databases \#2 and \#3 than for database \#1. Databases \#2 and \#3 only included publications that specified mosquito and virus species tested, sample size and detection technique (the full list of studies included in these databases is provided in Supplementary Table 1). A total of 32 and 16 factors were included in databases \#2 and \#3, respectively, and each unique combination of factors was considered a different entry (the full list of factors recorded is provided in Supplementary Table 2). Laboratory assays used for virus detection were divided into four broad categories. 'Animal' assays refer to the detection of pathological effects (including death) in laboratory animals, usually suckling mice, following inoculation with mosquito extracts; 'Cellular' assays refer to the detection of cytopathological effects in cell culture in vitro, following inoculation with mosquito extracts; 'Immunological' assays rely on the detection of viral antigens by antibodies (e.g., immunofluorescence assays) with or without previous amplification in cell culture or animal tissues; 'Molecular' assays refer to detection of viral nucleic acids, generally by reverse 
transcription (RT)-PCR. Whereas animal/cellular assays detect infectious virus and molecular assays detect viral nucleic acids (non-infectious), immunological assays include both detection of viral antigens in primary samples (non-infectious) and detection of viral antigens following amplification in vivo (e.g., intra-thoracic inoculation of Toxorhynchites mosquitoes) or in cell culture (infectious). Whether the use of infectious or non-infectious assays influenced the outcome of VT studies was also evaluated.

\subsection{Statistical analyses}

All statistical analyses were performed in the statistical environment R, version 3.0.2 (http://www.r-project.org/).

2.3.1. Historical trends. To normalize the overall scientific production about VT of dengue, West Nile and chikungunya viruses, the yearly number of publications between 1950 and 2013 was obtained through queries to the ISI Web of Science version 15.13.1 database, using the keywords 'dengue', 'West Nile virus' and 'chikungunya', respectively. For each virus, the normalized yearly number of publication was calculated as the ratio of the yearly number of publications about VT of this virus over the total yearly number of publications about the virus. The normalized yearly number of publications was analyzed using nonparametric Wilcoxon tests.

2.3.2. Evolution of virological assays. The evolution of laboratory assays used for VT detection in databases \#2 and \#3 was visualized by plotting the yearly proportion of each assay category (i.e., animal, cellular, immunological and molecular) over time. To weight each category according to the sample size, the yearly proportion of each assay category was calculated relative to the total number of individual mosquitoes tested for VT (i.e., the relative likelihood of each category over time). Smoothed curves were obtained by kernel density 
estimation using a Gaussian kernel and the bandwidth selection method proposed by Sheather and Jones (1991).

The association between assay category and VT detection probability was further explored with a subset of databases \#2 and \#3. The subset consisted of the Aedes-Flavivirus pair, which is the most frequent vector-virus association in the databases $(49.7 \%$ and $31.8 \%$ of entries in databases $\# 2$ and \#3, respectively). Analysis of the relationship between assay category and VT detection probability was restricted to the Aedes-Flavivirus pair to rule out the potential confounding effect of a differential VT probability among arbovirus families. Indeed, some arbovirus families may be vertically transmitted more efficiently than others (Turell, 1988). If VT of arboviruses with higher VT efficiency were predominantly examined using one particular virological assay, then a spurious association would be detected between this assay and higher VT detection probability. In experimental studies, only three assay categories were compared for the Aedes-Flavivirus pair because the molecular assay category was not represented in database \#2. VT occurrence was defined as the proportion of database entries that found evidence of VT. Evolution of VT detection over time for the AedesFlavivirus pair was represented as the relative likelihood of yearly VT occurrence. The relative likelihood function was estimated by kernel density estimation as described above.

To disentangle the respective effects of assay and sample size, VT occurrence was analyzed with a generalized linear model that included the $\log _{10}$-transformed sample size, the assay category and their interaction. As VT occurrence is a binary variable $(0=\mathrm{VT}$ undetected, $1=\mathrm{VT}$ detected), the model was fitted with a binomial error distribution and a logit link function. Statistical significance of the effects was assessed by analysis of deviance (Hastie and Pregibon, 1991). The same model was used to evaluate the influence of infectious compared to non-infectious assays. 
To compare sample sizes between assay categories, distributions of $\log _{10}$-transformed sample sizes for each assay category were compared with non-parametric Kruskall-Wallis tests. When significant, pairwise Wilcoxon tests were subsequently performed with a Bonferroni correction of the $p$-values. 


\section{Results and discussion}

\subsection{Summary description of databases}

The primary database (database \#1) includes 257 articles related to arbovirus VT published between 1899 and 2013 (the full list of publications is provided in Supplementary Table 1). Two additional databases were derived from database \#1 that were restricted to 'experimental' and 'natural' types of studies (database \#2 and \#3, respectively) and had more stringent inclusion criteria (see material and methods).

Database \#2 includes a total of 1,119 entries from 94 distinct publications that examined VT in an experimental setting. Overall, VT occurrence was $60.2 \%$ in database \#2 (i.e., evidence of VT was found in $60.2 \%$ of entries). Virological assays consisted of $58.5 \%$ immunological, $23.6 \%$ cellular, $16.8 \%$ animal and $1.1 \%$ molecular assays. The most frequent vector-virus pairs in database \#2 were Aedes-Flavivirus, Culex-Flavivirus, AedesOrthobunyavirus and Aedes-Alphavirus that are represented by $49.7 \%, 22.6 \% 18.9 \%$ and $5.1 \%$ of entries, respectively. The remaining $3.7 \%$ consist of 11 less studied vector-virus pairs. The median VT prevalence, estimated as the number of positive pools over the total number of mosquitoes tested, was $0.15 \%, 0.0 \%, 13.5 \%$ and $0.0 \%$ in Aedes-Flavivirus, CulexFlavivirus, Aedes-Orthobunyavirus and Aedes-Alphavirus pairs, respectively.

Database \#3 includes a total of 368 entries from 97 distinct publications that examined VT in a natural setting. Overall, VT occurrence was $37.5 \%$ in database \#3 (i.e., evidence of VT was found in $37.5 \%$ of the entries). Virological assays consisted of $29.6 \%$ animal, $28.5 \%$ immunological, 25.3\% cellular and 16.3\% molecular assays. Aedes-Flavivirus, CulexFlavivirus, Aedes-Orthobunyavirus and Aedes-Alphavirus pairs are represented by $31.8 \%$, $21.2 \% 16.8 \%$ and $2.7 \%$ of database \#3 entries, repectively. The remaining $27.5 \%$ consist of 19 less studied vector-virus pairs. The median VT prevalence was $0.03 \%, 0.0 \%, 0.0 \%$ and 
0.06\% in Aedes-Flavivirus, Culex-Flavivirus, Aedes-Orthobunyavirus and Aedes-Alphavirus pairs, respectively. The 10- to 1000 -fold lower estimates of VT prevalence observed in natural studies likely reflect two different processes. First, the infection prevalence in the previous adult generation is generally close to $100 \%$ in experimental studies because mothers are experimentally exposed to the arbovirus before VT is assayed in the their progeny. The prevalence of arbovirus infection in wild mosquito populations, by contrast, is typically low and the prevalence of VT infection observed in a given generation actually measures the product of adult population prevalence and VT efficiency. For example, only $0.1 \%$ of fieldcaught Ae. aegypti were found to be infected by dengue virus in an endemic region of Thailand (Yoon et al., 2012). Second, experimental VT studies possibly overestimate VT efficiencies because they tend to focus on naturally efficient combinations of vector-virus strains. VT efficiency may be further enhanced in experimental studies due to the unnatural mode of infection commonly used in the parental generation (i.e., intrathoracic inoculation). Moreover, VT efficiency can be quickly selected in the laboratory. For example, dengue virus VT efficiency increased more than 4-fold within two Ae. aegypti generations (Joshi et al., 2002). 'Stabilized' infections have also been described for arboviruses of the California serogroup (Bunyaviridae: Orthobunyavirus), such as California encephalitis virus in Ae. dorsalis (Turell et al., 1982) and San Angelo virus in Ae. albopictus (Tesh and Shroyer, 1980). Whereas TOT occurred in $10-20 \%$ of the progeny of horizontally infected females, transovarially infected daughters in the following generations consistently transmitted virus to over $90 \%$ of their progeny. The proposed mechanism is that following a systemic infection by horizontal transmission, only a small fraction of the developing oocytes of a female become infected transovarially. However, if the germinal cells of a female become infected, then nearly $100 \%$ of her progeny are infected. It has been argued that most experimental studies of TOT have in fact underestimated VT efficiency because they considered 'non-stabilized' 
infections (Tesh, 1984). An additional complicating factor is that TOT may not occur during the first gonotrophic cycles of orally infected females because the ovaries only become infected during the subsequent gonotrophic cycles. Only considering the first batch of eggs, usually free of virus, would also underestimate VT efficiency in this case (Tesh, 1984).

\subsection{Historical trends}

We first examined whether epidemiological landmarks, such as emergence events, large outbreaks or a significant change in geographic distribution of an arbovirus, influenced VT research. We considered three major epidemiological events in the recent history of arbovirology: the 1981 dengue hemorrhagic fever (DHF) epidemic in the Americas, the 1999 West Nile virus emergence in North America, and the 2005 chikungunya epidemic in the Indian Ocean region. Whereas dengue was already a growing problem in the tropics in the early 1980s (Messina et al., 2014), the emergence of West Nile virus in 1999 and that of chikungunya in 2005 were sudden and largely unexpected.

3.1.1. DHF in the Americas. Dengue viruses (Flaviviridae: Flavivirus) consist of four serotypes (DENV-1 to -4) that are currently estimated to collectively cause about 96 million symptomatic cases of dengue fever each year, including the severe form DHF (Bhatt et al., 2013). Dengue viruses are primarily transmitted by the mosquito Ae. aegypti, which was largely eliminated from Central and South America during the 1950s and 1960s by the eradication program of the Rockefeller Foundation. Approximately ten years after the end of the eradication program, however, dengue epidemics started to occur in the Americas, quickly followed by hyperendemicity and the emergence of DHF (Bennett et al., 2010). The first cases of DHF were reported in Cuba during a DENV-2 epidemic in 1981 (Guzmán et al., 1999). 
This epidemiological event did not appear to have an obvious impact on dengue research overall, as shown by a query with the keyword 'dengue' to the ISI Web of Science database (Fig 2.). By contrast, research on dengue virus VT markedly increased after 1981 (Fig. 2). The normalized yearly number of VT publications was significantly larger during the period 1982-2013 than during the period 1950-1981 (Wilcoxon test, $p=2.0 \times 10^{-8}$ ). We cannot exclude the existence of a concomitant factor, however, because this effect was no longer significant when considering a period spanning 5 years before and 5 years after the event (Wilcoxon test, $p=1.2 \times 10^{-1}$ ). The burst of VT research after the early $1980 \mathrm{~s}$ may have been driven by studies investigating the possibility of dengue virus maintenance in areas where Ae. aegypti re-established, following 'rediscovery' of arbovirus VT in the previous decade (i.e., a delayed consequence of the Watts et al. 1973 publication). Despite a lack of evidence in the early studies (Siler et al., 1926; Simmons et al., 1931), the VT hypothesis has been particularly popular in the case of dengue. Although a sylvatic transmission cycle exists whereby sylvatic dengue strains circulate between arboreal mosquitoes and non-human primates, there is no identified animal reservoir of dengue virus strains responsible for human epidemics (Vasilakis and Weaver, 2008). This probably explains why VT has often been suggested to explain dengue virus maintenance during inter-epidemic periods.

3.1.2. West Nile virus emergence in North America. West Nile virus (Flaviviridae: Flavivirus) is primarily transmitted between birds and ornithophilic mosquitoes but can occasionally infect humans and horses, which are dead-ends for the virus (World Health Organization, 2011). Following its introduction in North America, it is estimated that between 1999 and 2010, West Nile virus infected 1.8 million people, resulting in 1,308 deaths (Kilpatrick, 2011). Research on this virus clearly increased after the North-American emergence (Fig. 3) but the normalized yearly number of studies on West Nile virus VT also increased significantly (Fig. 3; Wilcoxon test, $p=6.1 \times 10^{-9}$ ). The effect was also significant 
when considering a period spanning 5 years before and 5 years after the event (Wilcoxon test, $p=3.7 \times 10^{-3}$ ). More frequent studies on West Nile virus VT in Culex mosquitoes after the 1999 epidemic have probably been stimulated by the search for a possible arthropod reservoir. Adult female mosquitoes are not active all year long in temperate regions of North America, yet overwintering persistence of West Nile virus transmission has been observed for over a decade (Reisen, 2013). The role of diapausing female Culex mosquitoes as a West Nile virus reservoir is one of the hypotheses to explain West Nile virus overwintering in North America (Reisen, 2013). Indeed, infected diapausing females have been reported in nature (e.g., Fechter-Leggett et al., 2012). VT is a prerequisite for this mechanism to operate, because Culex females do not blood feed prior to diapause (Mitchell and Briegel, 1989; Nelms et al., 2013). In warmer regions of North America, however, female Culex mosquitoes do not necessarily enter diapause and may instead become quiescent, sometimes after taking a blood meal (Eldridge, 1966; Nelms et al., 2013).

3.1.3. Chikungunya emergence in the Indian Ocean region. Chikungunya virus (Togaviridae: Alphavirus) is an arbovirus originating in Africa that primarily circulates between non-human primates and arboreal mosquitoes. In 2004, it emerged in the human population on the coast of Kenya and was subsequently responsible for a vigorous epidemic in the Indian Ocean region between 2005 and 2007. On La Réunion Island, 34.4\% of the population was infected overall, while 1.4 million cases were reported in 2006 in India (Pialoux et al., 2007). The global emergence of chikungunya that followed the 2005 outbreak triggered a renewed scientific interest for chikungunya virus, as shown by a query with the keyword 'chikungunya' to the ISI Web of Science database (Fig. 4). Although the normalized yearly number of publications on chikungunya virus VT after 2005 remains relatively low (due to the dramatic increase in the total number of chikungunya publications used for normalization), the frequency of VT publications significantly increased (Fig. 4; Wilcoxon 
test, $p=2.9 \times 10^{-3}$ ). The effect was also significant when considering a period spanning 5 years before and 5 years after the event (Wilcoxon test, $p=3.6 \times 10^{-2}$ ). Increase of VT research after the 2005 epidemic was, however, less dramatic than in the cases of the 1981 DHF epidemic in the Americas and the 1999 emergence of West Nile virus in North America. This may be a consequence of earlier studies, which concluded that Alphavirus VT rarely occurs and is unlikely to be epidemiologically significant when it does (Turell, 1988). Thus, the modest increase of research on chikungunya virus VT after 2005 may reflect a general lack of interest and/or a significant proportion of negative results, a well known bias in systematic reviews (Littell et al., 2008). The risk of chikungunya virus emergence in Europe, as illustrated by the Italian outbreak in 2007 (Rezza et al., 2007) and the current emergence in the Americas (Leparc-Goffart et al., 2014) may stimulate additional research on chikungunya virus VT.

\subsection{Evolution of virological assays}

For some authors (Bocquet and Molero, 1996), the expansion of arbovirus VT research in the 1970s may be related to the development of new investigation methods, notably with the introduction of immunological reactions in laboratory assays. Overall, virological assays used for VT detection changed dramatically over the last century (Fig. 5). In the 1970s, traditional animal models were replaced by immunological methods that became progressively more common through the 1990s. Immunological assays were then replaced by cellular and molecular assays in the 2000s. Although the use of animal, cellular and immunological assays followed a relatively similar evolution in both natural and experimental studies of arbovirus VT, molecular assays have seldom been used in experimental studies to date (Fig. 5A). 
The advent of novel virological methods such as PCR-based molecular assays may have facilitated VT detection due to improved sensitivity and higher-throughput capacity. It is worth noting that molecular assays only detect viral RNA and therefore do not provide evidence of infectious virus unless they are confirmed by virus isolation. This is also true for immunological assays that detect viral antigens without direct evidence for infectivity. VT being a relatively rare event (Bocquet and Molero, 1996), one might expect that the likelihood of VT detection would have increased with the evolution of assay performance. We tested this hypothesis in the subset of studies that examined Flavivirus VT in Aedes mosquitoes. The Aedes-Flavivirus association is the most widely represented in the published literature and has been investigated with a variety of laboratory assays. The probability of VT detection in Aedes-Flavivirus studies significantly increased over time, both in experimental (Fig. 6A) and natural studies (Fig. 6B). Although a causal relationship cannot be conclusively inferred, these increasing trends coincide with the advent of cellular and immunological assays in experimental studies (Fig. 5A) and of cellular and molecular assays in natural studies (Fig. 5B). Thus, our analyses support the hypothesis that the probability of VT detection has been influenced by the evolution of virological assays.

The probability of VT detection could have been influenced by the sensitivity of the assay and/or the ability to process a larger number of samples by modern assays. More recent detection methods such as cellular or immunological assays used in experimental studies are indeed associated with larger sample sizes (Fig. 7A; Kruskal-Wallis test, $p=5.2 \times 10^{-5}$; pairwise Wilcoxon tests: $p=6.4 \times 10^{-3}$ and $p=3.7 \times 10^{-5}$ for animal $v s$. cellular and animal $v s$. immunological assays, respectively, $p=6.3 \times 10^{-1}$ for cellular vs. immunological assays). Similarly, in natural studies, significantly larger sample sizes are found in studies based on molecular assays (Fig. 7B; Kruskal-Wallis test, $p=2.9 \times 10^{-2}$; pairwise Wilcoxon tests: $p=$ $2.6 \times 10^{-2}$ for animal $v s$. molecular assays, $p=9.5 \times 10^{-1}$ for animal $v s$. cellular assays, $p=2.3$ 
$\mathrm{x} 10^{-1}$ for animal $v s$. immunological assays, $p=1.0$ for immunological $v s$. cellular assays, $p=$ $2.5 \times 10^{-1}$ for molecular $v s$. cellular assays, and $p=9.6 \times 10^{-1}$ for molecular $v s$. immunological assays). To determine the respective contributions of sample size and detection assay to changes in VT detection probability, VT occurrence in the databases was analyzed with a model that incorporated both factors and their interaction. In experimental studies, we found that VT occurrence was statistically significantly influenced by both the $\log _{10}$-transformed sample size $\left(p=2.5 \times 10^{-7}\right)$ and the detection assay $\left(p=3.7 \times 10^{-2}\right)$, but not by their interaction $\left(p=4.7 \times 10^{-1}\right)$. Therefore, the increase in VT detection probability did not simply result from a higher-throughput capacity of assays but also from improved assay sensitivity. In natural studies, VT occurrence was significantly influenced by the $\log _{10}$-transformed sample size $\left(p=3.9 \times 10^{-4}\right)$, but not by the detection assay $\left(p=2.2 \times 10^{-1}\right)$ or their interaction $\left(p=7.2 \times 10^{-2}\right)$. In this case, the observed increase in VT detection probability primarily resulted from a significant increase in the number of samples tested.

We also evaluated whether the use of infectious or non-infectious assays influenced the probability of VT detection. Both animal and cellular assays detect infectious virus, whereas molecular assays detect viral nucleic acids (non-infectious). Immunological assays include both detection of viral antigens in primary samples (non-infectious) and detection of viral antigens following amplification in vivo or in cell culture (infectious). We did not find a statistically significant effect of whether the assay was infectious or non-infectious, both in experimental studies $\left(p=1.8 \times 10^{-1}\right)$ and in natural studies $\left(p=1.8 \times 10^{-1}\right)$, neither did we find an interaction with the $\log _{10}$-transformed sample size $\left(p=1.5 \times 10^{-1}\right.$ and $p=8.6 \times 10^{-2}$, respectively). 


\section{Conclusions}

Arbovirus VT in mosquitoes has rarely taken center stage in arbovirology, but has remained a debated topic since it was initially discovered more than a century ago. In the present study, fortunately, controversy about the extent and epidemiological importance of VT for arbovirus epidemiology has likely alleviated publication bias, a well-known pitfall of meta-analytical studies due to the under reporting of negative results. Despite their intrinsic value, negative results often remain unpublished because they are abandoned by authors and/or rejected by editors. Controversial topics like arbovirus VT do not entirely escape publication bias, but are thought to minimize it because negative results are more frequently published (e.g., Vazeille et al., 2009; Watts et al., 1985). By nature, however, publication bias is difficult to quantify. Therefore, caution must be used to interpret the results, keeping in mind that under reporting of negative results leads to overestimating VT.

Although the existence of VT is unequivocal for several mosquito-arbovirus pairs of public health significance, our databases showed that VT is collectively rare. Studies that estimated VT prevalence in a natural situation typically found that $<0.1 \%$ of mosquitoes are vertically infected, regardless of the virus family or mosquito species. VT efficiency per se, however, cannot be inferred in field studies because infection prevalence in the adult population is unknown. VT prevalence in experimental studies is 10 to 1000 -fold higher, which reflects more closely VT efficiency because the parental generation is usually $100 \%$ infected. Even when it is relatively efficient, VT is unlikely to explain the persistence of arboviruses in the prolonged absence of horizontal transmission. A recent mathematical model on dengue concluded that VT efficiency would have to be 5- to 30 -fold higher than the experimentally measured VT efficiency to support long-term persistence of the virus (Adams and Boots, 2010). It has been argued that TOT alone could maintain the virus indefinitely in a 
mosquito subpopulation whose germinal tissues are permanently infected (Turell et al., 1982) even if the prevalence in the overall population is low (Tesh, 1984). Unless VT efficiency is $100 \%$, however, occasional horizontal amplification is predicted to be necessary for the longterm maintenance of arboviruses (Fine, 1975).

Our historical perspective highlighted two important aspects of VT research in arbovirology. First, scientific production on arbovirus VT in mosquitoes has been extremely uneven over time. In particular, epidemiological landmarks such as emergence events have significantly stimulated VT research. Second, the evolution of virological assays used in VT studies has profoundly influenced the ability to detect it. Increased sensitivity and higherthroughput of more recent laboratory assays are associated with higher estimates of VT rates. Taken together, our results call for caution when interpreting VT studies because their conclusions are context- and method-dependent. 


\section{Acknowledgements}

The authors thank members of the Lambrechts lab for insightful discussions, Richard Paul for critical reading of the manuscript, and two anonymous reviewers for useful comments. S.L. was supported by a doctoral fellowship from University Pierre and Marie Curie. L.L. received funding from the French Government's Investissement d'Avenir program, Laboratoire d'Excellence Integrative Biology of Emerging Infectious Diseases (grant ANR10-LABX-62-IBEID). 


\section{References}

Adams, B., Boots, M., 2010. How important is vertical transmission in mosquitoes for the persistence of dengue? Insights from a mathematical model. Epidemics 2, 1-10.

Bennett, S.N., Drummond, A.J., Kapan, D.D., Suchard, M.A., Munoz-Jordan, J.L., Pybus, O.G., Holmes, E.C., Gubler, D.J., 2010. Epidemic dynamics revealed in dengue evolution. Mol. Biol. Evol. 27, 811-818.

Bhatt, S., Gething, P.W., Brady, O.J., Messina, J.P., Farlow, A.W., Moyes, C.L., Drake, J.M., Brownstein, J.S., Hoen, A.G., Sankoh, O., Myers, M.F., George, D.B., Jaenisch, T., Wint, G.R.W., Simmons, C.P., Scott, T.W., Farrar, J.J., Hay, S.I., 2013. The global distribution and burden of dengue. Nature 496: 504-507.

Bocquet, J.-P., Molero, M., 1996. Une hypothèse originale : la transmission transovarienne des arbovirus de Finlay à nos jours, in: Waast, R., Moulin, A.M. (Eds.). Presented at the Les sciences hors d'Occident au 20ème siècle - Médecines et Santé, Paris, pp. 83-88.

Centers for Disease Control and Prevention, 2010. Arbovirus Catalog [WWW Document]. wwwn.cdc.gov. URL http://wwwn.cdc.gov/Arbocat/Default.aspx (accessed 1.20.14).

Chamberlain, R.W., Kissling, R.E., Stamm, D.D., Nelson, D.B., Sikes, R.K., 1956a. Venezuelan equine encephalomyelitis in wild birds. Am. J. Hyg. 63, 261-273.

Chamberlain, R.W., Sikes, R.K., Nelson, D.B., 1956b. Infection of Mansonia perturbans and Psorophora ferox mosquitoes with Venezuelan equine encephalomyelitis virus. Proc. Soc. Exp. Biol. Med. 91, 215-216.

Chamberlain, R.W., Sudia, W.D., 1957. The North American arthropod-borne encephalitis viruses in Culex tarsalis Coquillett. Am. J. Hyg. 66, 151-159.

Chamberlain, R.W., Sudia, W.D., Gillett, J.D., 1959. St. Louis encephalitis virus in mosquitoes. Am. J. Hyg. 70, 221-236. 
Davis, N.C., Shannon, R.C., 1930. The location of yellow fever virus in infected mosquitoes and the possibility of hereditary transmission. Am. J. Epidemiol. 11, 335-344.

Diaz, L.A., Flores, F.S., Quaglia, A., Contigiani, M.S., 2012. Intertwined arbovirus transmission activity: reassessing the transmission cycle paradigm. Front. Physiol. 3, 493.

Ebert, D., 2013. The epidemiology and evolution of symbionts with mixed-mode transmission. Annu. Rev. Ecol. Evol. Syst. 44, 623-643.

Eldridge, B.F., 1966. Environmental control of ovarian development in mosquitoes of the Culex pipiens complex. Science 151, 826-828.

Fechter-Leggett, E., Nelms, B.M., Barker, C.M., Reisen, W.K., 2012. West Nile virus cluster analysis and vertical transmission in Culex pipiens complex mosquitoes in Sacramento and Yolo Counties, California, 2011. J. Vector Ecol. 37, 442-449.

Fine, P.E., 1975. Vectors and vertical transmission: an epidemiologic perspective. Ann. N. Y. Acad. Sci. 266, 173-194.

Finlay, C.J., 1899. Mosquitoes considered as transmitters of yellow fever and malaria. Med. Record 55, 737-739.

Frobisher, M., Jr, Davis, N.C., Shannon, R.C., 1931. On the failure of yellow fever virus to persist in a colony of Aedes aegypti. Am. J. Epidemiol. 14, 142-146.

Gubler, D.J., 2001. Human arbovirus infections worldwide. Ann. N. Y. Acad. Sci. 951, 1324.

Gubler, D.J., 2002. The global emergence/resurgence of arboviral diseases as public health problems. Arch. Med. Res. 33, 330-342.

Guiteras, J., 1901. Experimental yellow fever at the inoculation station of the Samtary Department of Havana with a view to producing immunization. Am. Med. 2, 809-817.

Guzmán, M.G., Kourí, G., Vázquez, S., Rosario, D., Bravo, J., Valdés, L., 1999. DHF epidemics in Cuba, 1981 and 1997: some interesting observations. Dengue Bull. 23, 39- 
43.

Hastie, J.T., Pregibon, D., 1991. Generalized linear models, in: Chambers, J.M., J, H.T. (Eds.), Statistical Models in S. Wadsworth \& Brooks/Cole.

Hindle, E., 1930. The transmission of yellow fever. Lancet 216, 835-842.

Joshi, V., Mourya, D.T., Sharma, R.C., 2002. Persistence of dengue-3 virus through transovarial transmission passage in successive generations of Aedes aegypti mosquitoes. Am. J. Trop. Med. Hyg. 67, 158-161.

Kilpatrick, A.M., 2011. Globalization, land use, and the invasion of West Nile virus. Science $334,323-327$.

Kissling, R.E., Chamberlain, R.W., Nelson, D.B., Stamm, D.D., 1956. Venezuelan equine encephalomyelitis in horses. Am. J. Hyg. 63, 274-287.

Kissling, R.E., Chamberlain, R.W., Sudia, W.D., Stamm, D.D., 1957. Western equine encephalitis in wild birds. Am. J. Hyg. 66, 48-55.

Lambrechts, L., Scott, T.W., 2009. Mode of transmission and the evolution of arbovirus virulence in mosquito vectors. Proc. Biol. Sci. 276, 1369-1378.

Leake, C.J., 1984. Transovarial transmission of arboviruses by mosquitoes, in: Mayo, M.A., Harrap, K.A. (Eds.), Vectors in virus biology. Academic Press, New-York \& London, pp. 63-91.

Leparc-Goffart, I., Nougairede, A., Cassadou, S., Prat, C., de Lamballerie, X., 2014. Chikungunya in the Americas. Lancet 383, 514.

Littell, J.H., Corcoran, J., Pillai, V., 2008. Systematic reviews and meta-analysis. Oxford Univ Press, New-York.

Marchoux, É., Simond, P.-L., 1905. La transmission héréditaire du virus de la fièvre jaune chez le Stegomyia fasciata. C. R. Soc. Biol. Paris 259-260.

Marchoux, É., Simond, P.-L., 1906. Etudes sur la fièvre jaune - Deuxième mémoire. Ann. 
Inst. Pasteur 20, 1-25.

Messina, J.P., Brady, O.J., Scott, T.W., Zou, C., Pigott, D.M., Duda, K.A., Bhatt, S., Katzelnick, L., Howes, R.E., Battle, K.E., Simmons, C.P., Hay, S.I., 2014. Global spread of dengue virus types: mapping the 70 year history. Trends Microbiol. 22, 138-146.

Mitamura, T., Kitaoka, M., Watanabe, S., Hosoi, T., Tenjin, S., Seki, O., Nagahata, K., Jo, K., Shimizu, M., 1939. Weitere Untersuchungen über die Übertragung der japanischen epidemischen enzephalitis durch Mücken. Trans. Soc. Pathol. Jpn. 29, 92-105.

Mitchell, C.J.J., Briegel, H., 1989. Fate of the blood meal in force-fed, diapausing Culex pipiens (Diptera: Culicidae). J. Med. Entomol. 26, 332-341.

Nelms, B.M., Macedo, P.A., Kothera, L., Savage, H.M., Reisen, W.K., 2013. Overwintering biology of Culex (Diptera: Culicidae) mosquitoes in the Sacramento valley of California. J. Med. Entomol. 50, 773-790.

Pialoux, G., Gaüzère, B.-A., Jauréguiberry, S., Strobel, M., 2007. Chikungunya, an epidemic arbovirosis. Lancet Infect. Dis. 7, 319-327.

Reed, W., Carroll, J., Agramonte, A., 1901. Experimental yellow fever. Am. Med. 2, 15-23.

Reeves, W.C., 2004. Overwintering of arboviruses, in: Epidemiology and control of mosquito-borne arboviruses in CA, 1943-1987. Calif. Mosq. Vector Control Assoc., Sacramento, California, pp. 357-382.

Reisen, W.K., 2013. Ecology of West Nile virus in North America. Viruses 5, 2079-2105.

Rezza, G., Nicoletti, L., Angelini, R., Romi, R., Finarelli, A.C., Panning, M., Cordioli, P., Fortuna, C., Boros, S., Magurano, F., Silvi, G., Angelini, P., Dottori, M., Ciufolini, M.G., Majori, G.C., Cassone, A., CHIKV study group, 2007. Infection with chikungunya virus in Italy: an outbreak in a temperate region. Lancet 370, 1840-1846.

Root, J.J., Oesterle, P.T., Nemeth, N.M., Klenk, K., Gould, D.H., McLean, R.G., Clark, L., Hall, J.S., 2006. Experimental infection of fox squirrels (Sciurus niger) with West Nile 
virus. Am. J. Trop. Med. Hyg. 75, 697-701.

Root, J.J., Oesterle, P.T., Sullivan, H.J., Hall, J.S., Marlenee, N.L., McLean, R.G., Montenieri, J.A., Clark, L., 2007. Fox squirrel (Sciurus niger) associations with West Nile virus. Am. J. Trop. Med. Hyg. 76, 782-784.

Rosen, L., 1988. Further observations on the mechanism of vertical transmission of flaviviruses by Aedes mosquitoes. Am. J. Trop. Med. Hyg. 39, 123-126.

Rosenau, M.J., Goldberger, J., 1906. Attemps to grow the yellow fever parasite - The hereditary transmission of the yellow fever parasite in the mosquito. Yellow Fever Institute, Bulletin 15, 103-115.

Sheather, S.J., Jones, M.C., 1991. A reliable data-based bandwidth selection method for kernel density estimation. J. Roy. Stat. Soc. B 53, 683-690.

Siler, J.F., Hall, M.W., Hitchens, A.P., 1926. Dengue: its history, epidemiology, mechanism of transmission, etiology, clinical manifestations, immunity, and prevention. Philippine J. Sci. $29,1-322$.

Simmons, J.S., St John, J.H., Reynolds, F.H.K., 1931. Experimental studies of dengue. Philippine J. Sci. 44, 1-255.

Tesh, R.B., 1984. Transovarial transmission of arboviruses in their invertebrate vectors, in: Harris, K.F. (Ed.), Current topics in vector research. New York, pp. 57-76.

Tesh, R.B., Chaniotis, B.N., Johnson, K.M., 1972. Vesicular stomatitis virus (Indiana serotype): transovarial transmission by phlebotomine sandlies. Science 175, 1477-1479.

Tesh, R.B., Shroyer, D.A., 1980. The mechanism of arbovirus transovarial transmission in mosquitoes: San Angelo virus in Aedes albopictus. Am. J. Trop. Med. Hyg. 29, 1394.

Turell, M.J., 1988. Horizontal and vertical transmission of viruses by insect and tick vectors., in: Monath, T.P. (Ed.), The arboviruses: epidemiology and ecology. CRC Press, Boca Raton, Florida, pp. 127-152. 
Turell, M.J., Hardy, J.L., Reeves, W.C., 1982. Stabilized infection of California encephalitis virus in Aedes dorsalis, and its implications for viral maintenance in nature. Am. J. Trop. Med. Hyg. 31, 1252-1259.

Vasilakis, N., Weaver, S.C., 2008. The history and evolution of human dengue emergence. Adv. Virus Res. 72, 1-76.

Vazeille, M., Mousson, L., Failloux, A.-B., 2009. Failure to demonstrate experimental vertical transmission of the epidemic strain of chikungunya virus in Aedes albopictus from La Réunion Island, Indian Ocean. Mem. Inst. Oswaldo Cruz 104, 632-635.

Watts, D.M., Harrison, B.A., Pantuwatana, S., Klein, T.A., Burke, D.S., 1985. Failure to detect natural transovarial transmission of dengue viruses by Aedes aegypti and Aedes albopictus (Diptera: Culicidae). J. Med. Entomol. 22, 261-265.

Watts, D.M., Pantuwatana, S., DeFoliart, G.R., Yuill, T.M., Thompson, W.H., 1973. Transovarial transmission of LaCrosse virus (California encephalitis group) in the mosquito, Aedes triseriatus. Science 182, 1140-1141.

World Health Organization, 2011. West Nile virus [WWW Document]. World Health Organization. URL http://www.who.int/mediacentre/factsheets/fs354/en/ (accessed 3.8.14).

Yoon, I.-K., Getis, A., Aldstadt, J., Rothman, A.L., Tannitisupawong, D., Koenraadt, C.J.M., Fansiri, T., Jones, J.W., Morrison, A.C., Jarman, R.G., Nisalak, A., Mammen, M.P., Thammapalo, S., Srikiatkhachorn, A., Green, S., Libraty, D.H., Gibbons, R.V., Endy, T., Pimgate, C., Scott, T.W., 2012. Fine scale spatiotemporal clustering of dengue virus transmission in children and Aedes aegypti in rural Thai villages. PLoS Negl. Trop. Dis. 6, e1730. 


\section{Figure Legends}

Figure 1. Evolution of the yearly number of publications on arbovirus VT by mosquitoes. Bars show the yearly number of publications in each of five broad categories represented by different colors. The dashed vertical line indicates the publication of an influential Science article on LaCrosse virus VT by Watts et al. in 1973.

Figure 2. Evolution of the yearly number of publications on dengue virus VT. Bars represent the normalized number of yearly publications on dengue virus VT. The red curve shows the overall number of dengue publications used for normalization. The vertical dashed line indicates the start of the $1981 \mathrm{DHF}$ epidemic in the Americas.

Figure 3. Evolution of the yearly number of publications on West Nile virus VT. Bars represent the normalized number of yearly publications on West Nile virus VT. The red curve shows the overall number of West Nile virus publications used for normalization. The vertical dashed line indicates the start of the 1999 West Nile virus emergence in North America.

Figure 4. Evolution of the yearly number of publications on chikungunya virus VT. Bars represent the normalized number of yearly publications on chikungunya virus VT. The red curve shows the overall number of chikungunya publications used for normalization. The vertical dashed line indicates the start of the 2005 chikungunya epidemic in the Indian Ocean region.

Figure 5. Evolution of virological assays used for arbovirus VT detection in (A) experimental and (B) natural studies. In each panel, colored areas represent the relative 
likelihood of each assay category at a given time-point on the $x$-axis. The yearly proportion of each assay is calculated relative to the total number of individual mosquitoes tested for VT.

\section{Figure 6. Evolution of VT detection in (A) experimental and (B) natural studies of the} Aedes-Flavivirus pair. In each panel, the curve represents the relative likelihood of VT occurrence at a given time-point on the $x$-axis. The yearly detection rate is calculated relative to the total number of database entries.

Figure 7. Distribution of sample sizes by assay category in (A) experimental and (B) natural studies of arbovirus VT. Sample size was $\log _{10}$-transformed prior to analysis and graphical representation. Boxplots represent the $75^{\text {th }}$ percentile, median and $25^{\text {th }}$ percentile; whiskers extend to the highest and lowest value in the $1.5 \mathrm{x}$ interquartile range. 


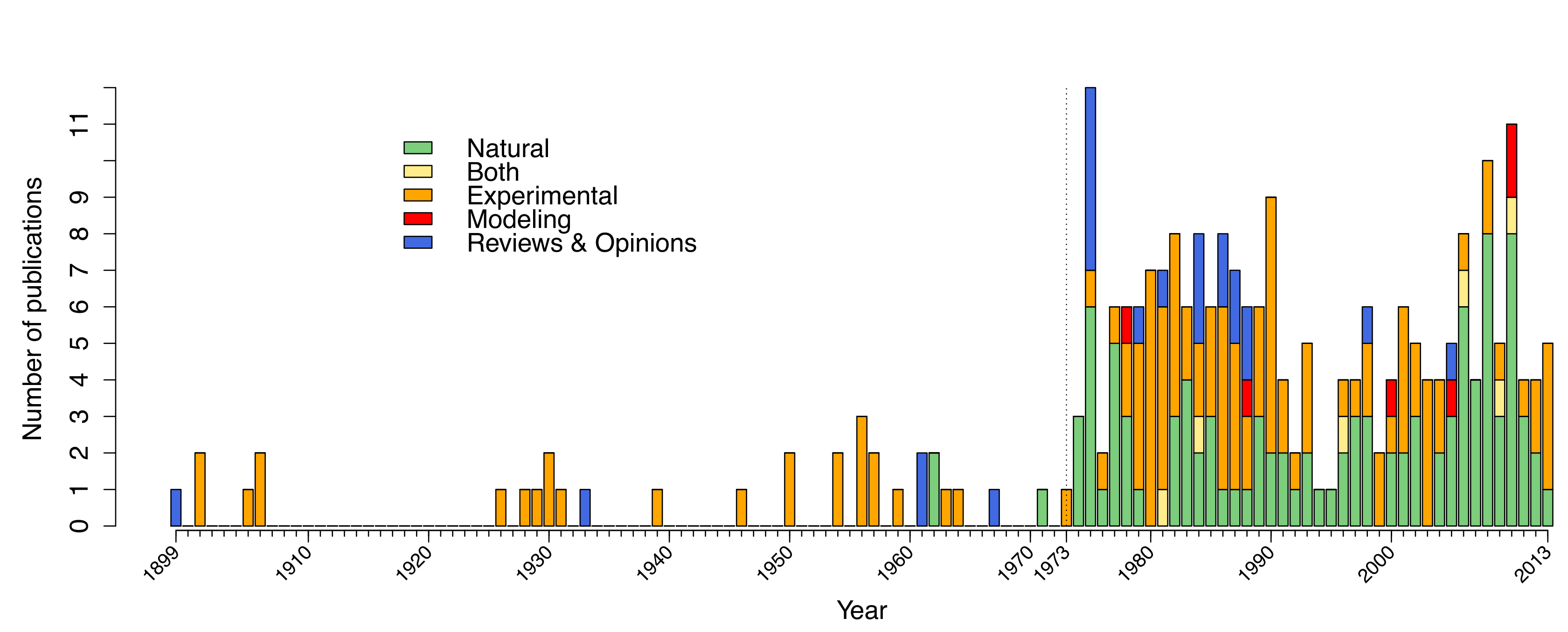




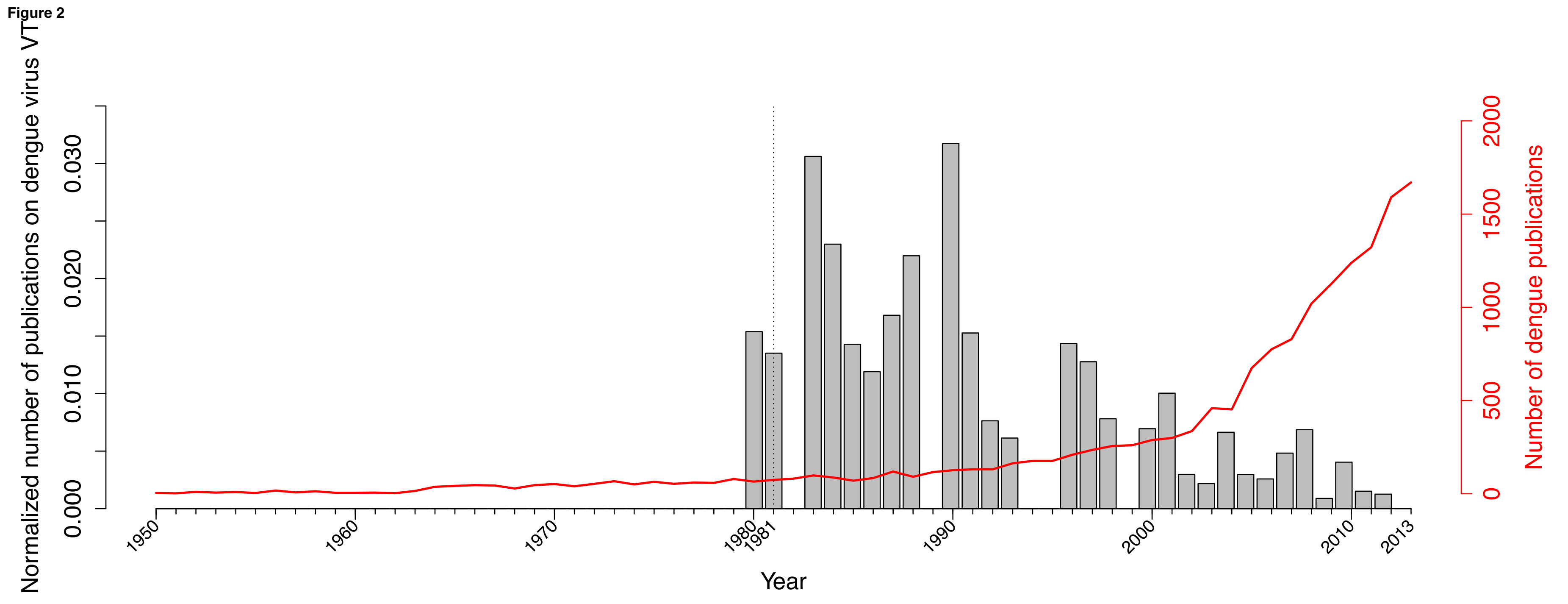

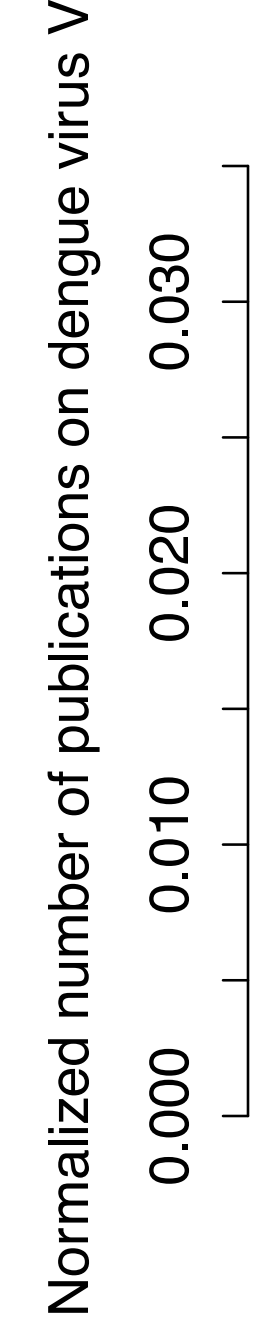

오․

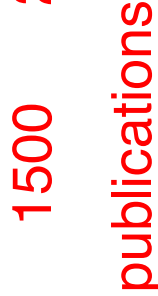

응 음

$\frac{4}{0}$
$\frac{1}{\Phi}$
$\frac{0}{2}$
$\frac{1}{5}$
$\frac{1}{z}$ 


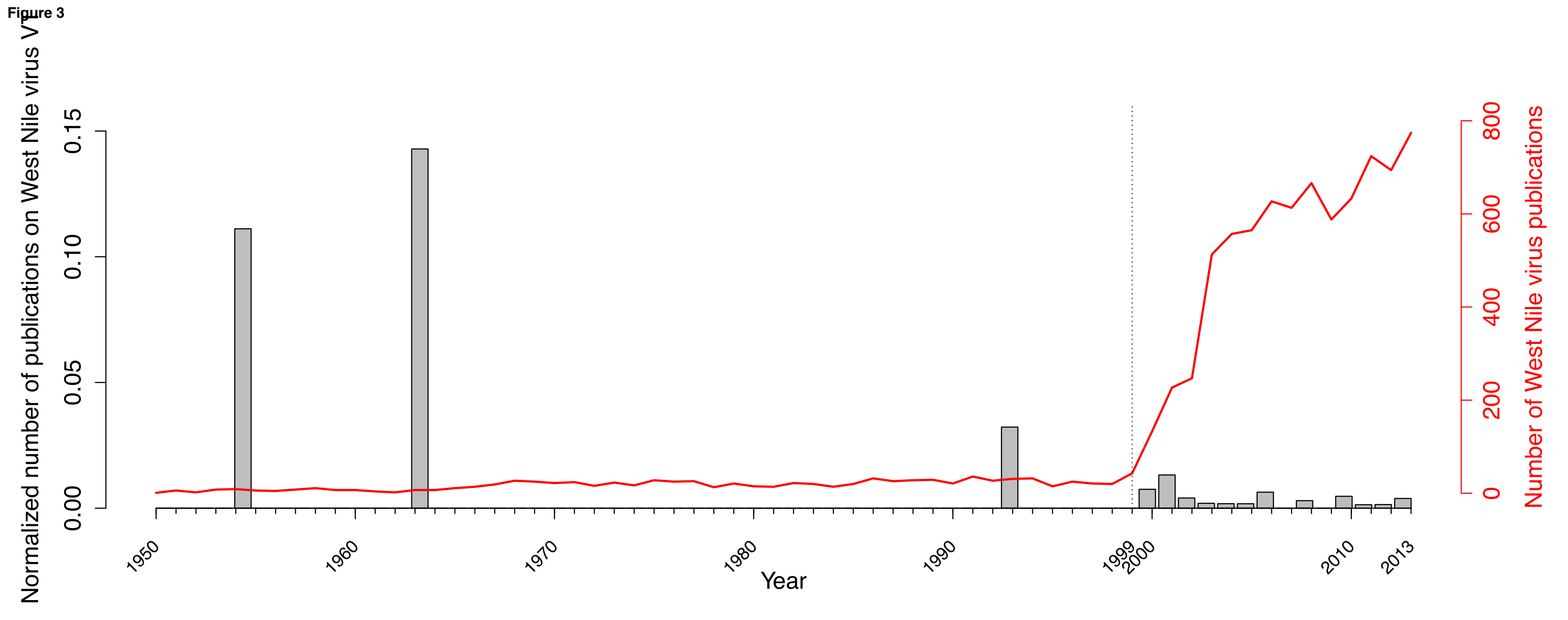




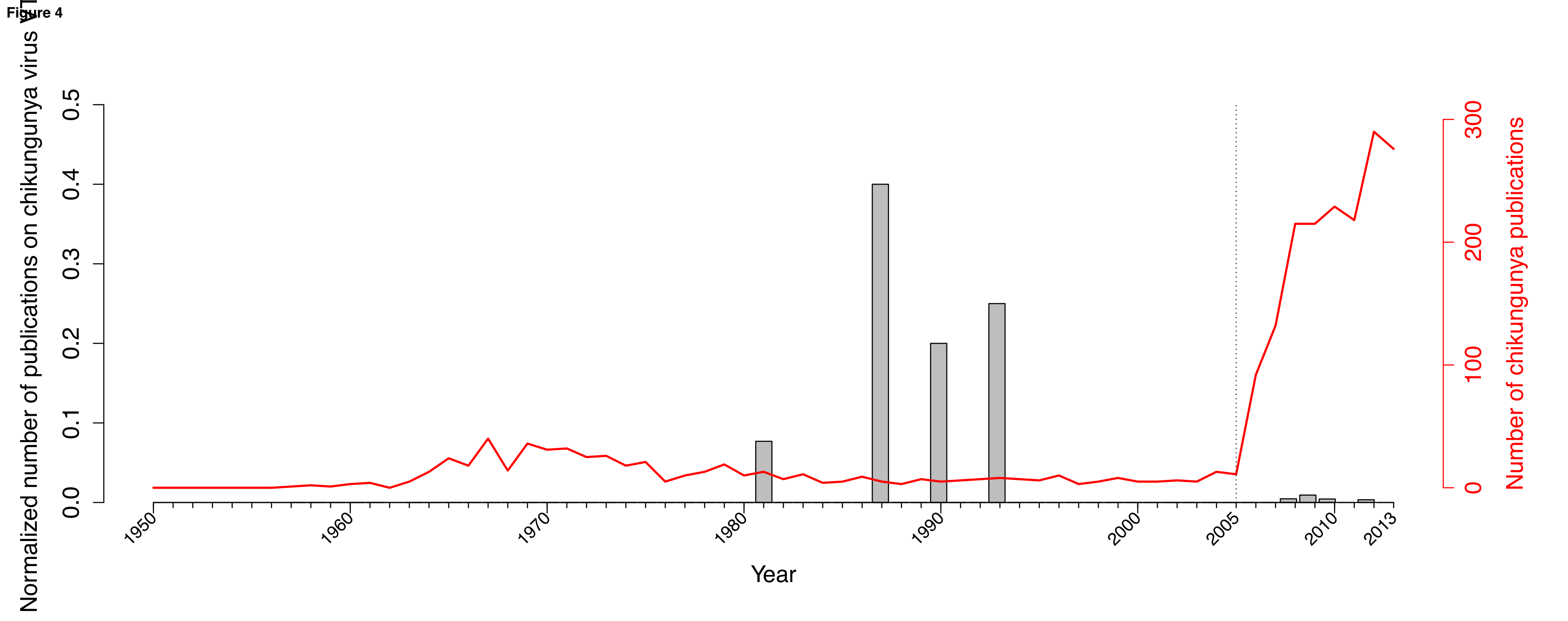


